\title{
Training for Bronchoscopists: Is Less More?
}

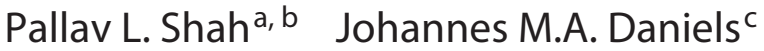 \\ ${ }^{a}$ NIHR Respiratory Biomedical Research Unit at the Royal Brompton and Harefield NHS Foundation Trust, Imperial \\ College, and ${ }^{b}$ Chelsea and Westminster Hospital NHS Foundation Trust, London, UK; ${ }^{c}$ VU University Medical Center, \\ Amsterdam, The Netherlands
}

Over the past 10 years, the complexity of bronchoscopy has dramatically evolved. Standard bronchoscopy consists of visual inspection with possible sampling by bronchial lavage, bronchial biopsy or bronchial brushings. Tumour resection and stent insertion were the domain of the few interventional pulmonologists that performed rigid bronchoscopy or the thoracic surgeons. In the last 10 years, there has been an increase in interventional procedures that are safely and easily performed under local anaesthesia with flexible bronchoscopy: from diathermy, argon plasma photocoagulation, cryotherapy through to stent insertion [1]. Furthermore, there has been the introduction of a number of lung volume reduction procedures for emphysema performed by flexible bronchoscopy utilising endobronchial valves [2-4] or endobronchial coils [5].

Procedures like airway bypass - although performed by flexible bronchoscopy - are very complex and involve several different skills such as the identification of blood vessels with ultrasound, transbronchial needle insertion, balloon dilatation and stent insertion [6]. Other procedures such as bronchial thermoplasty for asthma, however, require a pragmatic systemic approach and good anatomical knowledge to ensure that all of the possible accessible airways are treated with radiofrequency energy $[7,8]$.

These procedures are still the domain of selected bronchoscopists who have developed the new speciality of 'in-
(C) 2015 S. Karger AG, Basel

0025-7931/15/0893-0190\$39.50/0 terventional pulmonology'. The real challenge has been the rapid and widespread uptake of endobronchial ultrasound procedures. This procedure requires different skill sets, such as standard bronchoscopy, and success is based on the knowledge of the extrabronchial vasculature and bronchial and lymph node anatomy. Subtle differences are bronchoscope handling, for example the need to oppose the bronchoscope to the airway surface to obtain adequate ultrasound visualisation. For standard bronchoscopy, the standard teaching is still to avoid airway contact as this induces cough. The sampling process is also different from standard bronchoscopy. A study by Kemp et al. [9] demonstrated that different individuals acquire skills at different rates. In their study, the diagnostic outcomes for endobronchial ultrasound procedures performed by five different operators were evaluated for their first 100 procedures. It was evident that some individuals were competent in performing procedures very quickly, whereas others were still on their learning curve even after almost 100 procedures. Even though the operators in this study received no formal training and were early adopters of the technology, this study enforces the point that patients should not be exposed to risks and procedural failures due to individuals still in the training phase for these particular procedures. Whilst the apprenticeship model was the accepted method for skills training in the past, at present we agree that

\section{KARGER 125}

E-Mail karger@karger.com

www.karger.com/res
Pallav L. Shah

Royal Brompton Hospital

Sydney Street

London SW3 6NP (UK)

E-Mail pallav.shah@imperial.ac.uk 
patients should not bear the burden of the steep initial part of the learning curve. Simulation-based training is an ideal alternative because many skills can be obtained away from the bedside. A meta-analysis has shown that simulation-based bronchoscopy training is associated with large improvements in skills and behaviour [10].

Many different simulation settings exist nowadays, from plastic models to animal models through to virtual reality simulators. The available simulators all have slightly different purposes. For basic bronchoscopy, individuals need to gain anatomical knowledge and also learn how to handle and manipulate a bronchoscope. Plastic models such as Dexter allow the development of bronchoscope handling and dexterity [10]. Silicone models (e.g. Broncho Boy) provide a more realistic model of the bronchial tree and facilitate learning of the anatomy. They facilitate the development of dexterity specifically for the bronchial tree. Repeated procedures using such models help build muscle memory and improve the performance of the procedure. The virtual reality simulators have the advantage of recreating some real-life effects of bronchial secretions, anatomical variations, mucosal abnormalities, pathological findings and even coughing. They also provide feedback as the system can register parameters such as wall collisions and airways examined. Several reports have since shown that virtual reality simulation can be an effective teaching tool, although superiority over other simulation models has not been proven [11]. In the latest issue of Respiration, Colella et al. [12] go one step further by evaluating motion analysis of the bronchoscopist during a simulated flexible bronchoscopy. The authors state that only small movements in the bronchoscopist's left hand are necessary for adequate navigation through the patient's airways. In other words, less movement gives the bronchoscopist more control during the procedure. This and other statements frequently used in bronchoscopy training, such as 'slower is faster' and the 'the bronchoscope wants to do the bronchoscopy', all reflect the meticulous ergonomic design of the modern-day bronchoscope and the importance of adequate skills. The authors are worried that unsupervised simulation might result in wrong techniques and aimed to assess whether automated motion detection can detect differences between bronchoscopists. The study shows that motion analysis can discriminate between different experience levels. Interestingly, there was a strong negative correlation between the amount of deviation from the straight bronchoscope and the simulator score. This is a reassuring finding since it is apparently unlikely that students obtain a high simulator score with a poor handling technique. However, integration of automated motion detection in bronchoscopy simulators might enable real-time motion feedback during simulation and thereby improve the performance of bronchoscopy simulators in the future. Randomized trials are required to determine whether such high-tech simulators in combination with motion assessment of the operator yield better results than more simple simulation settings, also taking cost-effectiveness into account. A continuing international effort by the respiratory community should ultimately deliver better-skilled novices to the bedside.

\section{References}

$>1$ Toma T, Geddes DM, Shah PL: Brave new world of interventional bronchoscopy. Tho$\operatorname{rax} 2005 ; 60: 180-181$.

$\checkmark 2$ Sciurba FC, Ernst A, Herth FJ, et al: A randomized study of endobronchial valves for advanced emphysema. N Engl J Med 2010; 363:1233-1244.

$\checkmark 3$ Herth FJ, Noppen M, Valipour A, et al: Efficacy predictors of lung volume reduction with Zephyr valves in a European cohort. Eur Respir J 2012;39:1334-1342.

4 Shah PL, Herth FJF: Current status of bronchoscopic lung volume reduction with endobronchial valves. Thorax 2014;69:280-286.

5 Shah PL, Zoumot Z, Singh S, et al: Endobronchial coils for the treatment of severe emphysema with hyperinflation (RESET): a randomised controlled trial. Lancet Respir Med 2013;1:233-240.
-6 Shah PL, Slebos DJ, Cardoso PFG, et al: Bronchoscopic lung volume reduction with Exhale airway stents for emphysema (EASE trial): randomised, sham-controlled multicentre trial. Lancet 2011;378:997-1005.

$>7$ Castro M, Rubin AS, Laviolette M, et al: Effectiveness and safety of bronchial thermoplasty in the treatment of severe asthma: a multicenter, randomized, double-blind, sham-controlled clinical trial. Am J Respir Crit Care Med 2010;181:116-124.

8 Wechsler ME, Laviolette M, Rubin AS, et al: Bronchial thermoplasty: long-term safety and effectiveness in patients with severe persistent asthma. J Allergy Clin Immunol 2013;132: 1295-1302.
$>9$ Kemp SV, El Batrawy SH, Harrison RN, et al: Learning curves for endobronchial ultrasound using cusum analysis. Thorax 2010;65: 534-538.

10 Agro F, Sena F, Lobo E, et al: The Dexter Endoscopic Dexterity Trainer improves fibreoptic bronchoscopy skills: preliminary observations. Can J Anesth 2005;52:215-216.

11 Kennedy CC, Maldonado F, Cook DA: Simulation-based bronchoscopy training: systematic review and meta-analysis. Chest 2013; 144:183-192.

12 Colella S, Søndergaard Svendsen MB, Konge L, Svendsen LB, Sivapalan P, Clementsen P: Assessment of competence in simulated flexible bronchoscopy using motion analysis. Respiration 2015;89:155-161. 